\title{
Surface instability of soft films with coupled tension-shear interactions
}

\author{
Vijay Shenoy* \\ Materials Research Centre, Indian Institute of Science, Bangalore 560 012, India \\ Ashutosh Sharma^ \\ Department of Chemical Engineering, Indian Institute of Technology, Kanpur UP 208 016, India
}

\begin{abstract}
The surface roughening instability in a soft thin film, induced by interactions with a contactor is investigated accounting for both tension and shear effects in the interaction potential. A key finding of this study is that, in the presence of coupled tension-shear interactions, the instability mode will depend on whether the contactor is brought towards the film (contact mode) or the contactor is retracted from a fully sticking configuration (peeling mode). In the contact mode, the wavelength of the roughening is about three times the film thickness, in agreement with previous works, while in the peeling mode it is about four times the film thickness. Although the analysis indicates a rich and complex dependence on the parameters of the interaction, the wavelength of the instability is essentially independent of the interactions for physically reasonable values of interaction parameters. The results are in agreement with available experiments. The results of the present study are useful in providing insights into the physics of friction and cavitation at soft interfaces.
\end{abstract}

\section{INTRODUCTION}

Instabilities in thin films have been the focus of many recent investigations since they offer an attractive route to the creation of patterns at the micro/nanoscales. Both solid and liquid films are prone to instabilities due to the existence of several competing energies of comparable magnitude. For example, epitaxially grown solid films are prone to dislocation patterns, surface morphological instabilities, compositional instabilities due to competition between the elastic strain energy and the surface energy. ${ }^{1-5}$ Pattern formation via instabilities are also common in liquid films, for example, a liquid film can undergo spinodal dewetting due to a competition between excess intermolecular interactions and the surface energy, ${ }^{6-8}$ or due to external fields. ${ }^{9}$ The main feature of such instabilities is that the dominant wavelength of the patterns that emerge are strongly dependent on the nature and magnitude of the interactions.

Recent experiments ${ }^{10-12}$ have demonstrated that the surfaces of soft elastic (solid) films undergo a spontaneous roughening when brought in proximity of a second contacting surface. A key feature of this instability, in contrast to those reported earlier in solid and liquid films, ${ }^{1-9}$ is that, although conditions required to trigger the instability depend on the interactions, the wavelength of the patterns that emerge are independent of the nature of interactions. Indeed, the wavelength depends nearly linearly on the thickness of the film regardless of the surface properties and the mechanical properties of the system. The theory of this instability was presented in Refs. 13 and 14 where it was argued that the surface roughening is due to a competition between at-

*Author to whom correspondence should be addressed; electronic mail: shenoy@mrc.iisc.ernet.in

^Electronic mail: ashutos@iitk.ac.in tractive surface interactions (such as van der Waals and/or electrostatic) that favor a rough film, and the elastic strain energy in the film which inhibits surface roughening. It was shown that the surface roughening occurs at a critical separation distance or force, but the dominant wavelength of the pattern that emerges is independent of the interactions and depends linearly on the film thickness.

Although the theory presented in Refs. 13 and 14 explains the origin of the instability, and the linear dependence of the wavelength of the patterns to the thickness of the films, there are several aspects of the experiments in Refs. 10 and 11 that require further attention. An important point to be noted is that experiments in Ref. 10 are "peeling" experiments, i.e., the contacting glass slide initially is in near perfect adhesion with the film. The contacting glass slide is then "peeled" out, in a manner similar to propagating a crack along the interface between the film and the contacting slide. On the other hand, experiments in Ref. 11 are "contact" type experiments where the contactor is initially separated from the film surface. Contact is established by bringing the contractor to the proximity of the film, in the process of which patterns are formed. Although the wavelength of the patterns formed in both the experiments scales linearly with film thickness, the wavelength is about four times the film thickness in Ref. 10, and about three times in Ref. 11. There seems to exist, therefore, a difference in the nature of instabilities of the peeling experiment ${ }^{10}$ and the contact experiment. ${ }^{11}$

The point of departure of the present article from the analysis of Refs. 13 and 14 is in reference to the nature of the interaction potential. The analysis of Refs. 13 and 14 postulates an interaction potential between the contactor and the film surface that depends only on the normal gap thickness (indicated by $d_{z}$ in Fig. 2). While this assumption is physically meaningful in the case of contact type experiments, the 


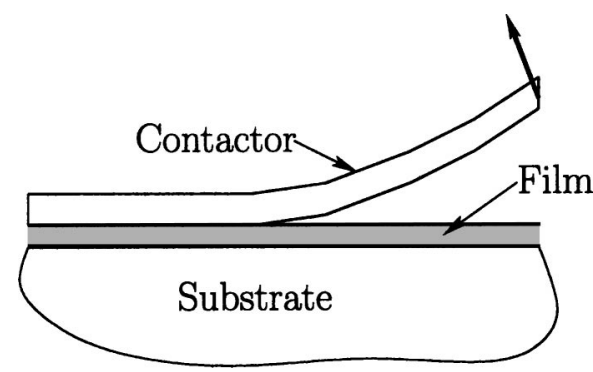

FIG. 1. Schematic of a peeling experiment. The thick arrow shows the direction of applied forces. Such an experiment will require the consideration of the couple tension-shear interactions between the film surface and the contactor.

following argument shows its limitations in peeling type experiments. The contactor is initially considered in perfect contact configuration with the film; it is now displaced parallel to the film surface (by an amount $d_{x}$ as shown in Fig. 2, keeping $d_{z}=0$ ). By the assumption of Refs. 13 and 14 that the interaction potential depends only on the normal displacement of the film surface, it is evident that this process will not any cost energy. Again, consider the peeling type of experiment where the contactor is retracted from the film surface with which it is initially in perfect contact (see Fig. 1). Clearly this type of experiment will include effects of the tangential displacement (shear displacement) between the curved contactor and the film surface. It is therefore evident that the assumption regarding the dependence of the interaction energy on only the normal gap thickness is inadequate to describe peeling type experiments. It is therefore necessary to include coupled tension-shear effects in the interaction potential, especially to describe the peeling experiments.

The importance of coupled tension-shear interaction in interfacial debonding processes has been previously illustrated by several workers. The influence of tension-shear coupled interactions on nucleation of dislocations at crack tips was studied in Ref. 15 . Interfacial debonding ${ }^{16}$ has been studied using cohesive interactions with coupled tensionshear, and later extended to the study of brittle fracture in solids. ${ }^{17}$ These works have used the universal binding energy type relations ${ }^{18}$ to describe the interfacial cohesion modified to account for shear displacements. Several workers have investigated the effect of cohesive interactions on adhesion (see, for example, Ref. 19 and references therein).

The aim of the present article is to develop a theory for surface instability induced by interactions accounting for both tension and shear effects. The article has two main sections: Section II, which presents the formulation, and Sec. III which contains the results of the analysis followed by a concluding section. The results presented here are likely to be useful in understanding a wide class of phenomena involving soft interfaces such as the peeling of adhesives, ${ }^{20}$ wafer debonding, etc. ${ }^{21}$

\section{FORMULATION}

We consider a thin solid film of thickness $h$ made of an incompressible linearly elastic material with shear modulus $\mu$, perfectly bonded to a substrate. The free surface of the

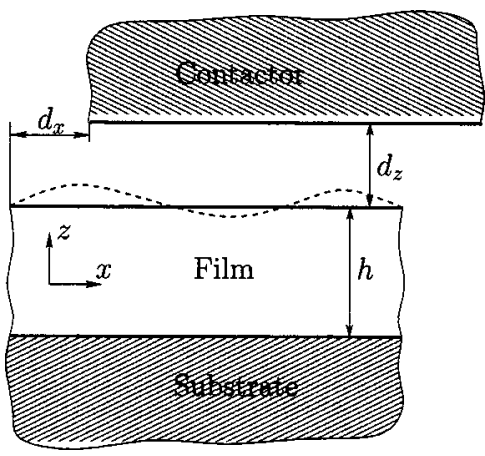

FIG. 2. A soft film interacting with a contactor. Material points in the film displace by $u$ in the $x$ direction and $w$ in the $z$ direction. Adapted from Ref. 14.

film is in proximity to a contactor as shown in Fig. 2. Both the contactor and the substrate are treated as rigid. The points in the film are described by a coordinate system as shown in Fig. 2 with $z=0$ being the free surface of the undisturbed film.

Under the action of the interactions with the contactor, the film undergoes elastic deformations described by displacement fields $u(x, z)$ and $w(x, z)$ in the $x$ and $z$ directions, respectively. The present study, therefore, restricts attention to plane strain deformations of the film. For any given experimental conditions (such as the value of the nominal gap thickness $d_{z}$ and shearing displacement $d_{x}$ as shown in Fig. 2 ), the equilibrium displacement field extremizes the potential energy of the system

$$
\Pi=\int_{V} W(\boldsymbol{\epsilon}) d V-\int_{S} U\left(\left|d_{x}-u_{s}\right|, d_{y}-w_{s}\right) d S,
$$

where $u_{s}$ and $w_{s}$ are values of the surface displacements, i.e., $u_{s}(x)=u(x, 0)$ and $w_{s}(x)=w(x, 0)$. Elastic strain energy density is denoted by $W(\boldsymbol{\epsilon})$ and is related to strain tensor $\boldsymbol{\epsilon}$ and the shear modulus $\mu$ in a well known way $^{22}$ and $U\left(u_{s}, w_{s}\right)$ is the potential of interaction between the free surface of the film and that of the contactor. The key physics included in the present work, in contrast to Refs. 13 and 14, is that the interaction potential is allowed to depend on the tangential surface displacement $u_{s}$, in addition to the normal surface displacement $w_{s}$ (which was the only dependence considered in Refs. 13 and 14). The interaction potential depends parametrically on $d_{x}$ and $d_{z}$. It is assumed to obey the symmetry condition

$$
U\left(-\left(d_{x}-u_{s}\right), d_{y}-w_{s}\right)=U\left(d_{x}-u_{s}, d_{y}-w_{s}\right)
$$

and hence denoted as $U\left(\left|d_{x}-u_{s}\right|, d_{y}-w_{s}\right)$.

\section{A. The homogeneous state}

For a given value of the nominal gap distance $d_{z}$ and shear displacement $d_{x}$ of the contactor, there exists a state such that the stresses are equal everywhere in the film, i.e., the film is flat and "unpatterned." The displacements $u$ is such that the shear strain is constant in the film and $w$ vanishes everywhere in the film. This owes to the fact the film is incompressible. In such a case, the stresses are described by a homogeneous pressure field given by 


$$
p^{h}(x, z)=F_{z},
$$

where

$$
F_{z}=\left.\frac{\partial U}{\partial w_{s}}\right|_{w_{s}=0}
$$

and a shear stress field

$$
\sigma_{x z}^{h}=\frac{\mu}{h} u_{s}=\left.\frac{\partial U}{\partial u_{s}}\right|_{w_{s}=0}=F_{x} .
$$

The last equation is an implicit equation for surface shear displacement $u_{s}$ of the film. Evidently, the values of $F_{x}$ and $F_{z}$ depend on the nominal gap thickness $d_{z}$ and the shear displacement $d_{x}$ of the contactor, along with other parameters that determine the interaction potential. The homogeneous displacement field is given by

$$
u^{h}(x, z)=u_{s}^{h}\left(1+\frac{z}{h}\right), \quad w^{h}(x, z)=0,
$$

where $u_{s}^{h}$, as mentioned above, is the solution of Eq. (5).

\section{B. Stability analysis}

The stability of the homogeneous state is investigated by perturbing the homogeneous state [with displacements given in Eq. (6)] by displacement fields $u_{s}^{p}$ and $w_{s}^{p}$. The interaction potential is expanded in a series in $u_{s}^{p}$ and $w_{s}^{p}$ about the homogeneous state

$$
\begin{aligned}
U\left(u_{s}^{h}+u_{s}^{p}, w_{s}^{h}+w_{s}^{p}\right)= & U\left(u_{s}^{h}, w_{s}^{h}\right)+F_{x} u_{s}^{p}+F_{z} w_{s}^{p} \\
& +\frac{1}{2}\left[\mathcal{Y}_{s}\left(u_{s}^{p}\right)^{2}+2 \mathcal{Y}_{\mathrm{st}} u_{s}^{p} w_{s}^{p}\right. \\
& \left.+\mathcal{Y}_{t}\left(w_{s}^{p}\right)^{2}\right]+O\left(u_{s}^{3}, w_{s}^{3}\right),
\end{aligned}
$$

where

$$
\begin{aligned}
& \mathcal{Y}_{s}=\left.\frac{\partial^{2} U}{\partial u_{s}^{2}}\right|_{u_{s}=u_{s}^{h}, w_{s}=w_{s}^{h}}, \\
& \mathcal{Y}_{s t}=\left.\frac{\partial^{2} U}{\partial u_{s} \partial w_{s}}\right|_{u_{s}=u_{s}^{h}, w_{s}=w_{s}^{h}}, \\
& \mathcal{Y}_{t}=\left.\frac{\partial^{2} U}{\partial w_{s}^{2}}\right|_{u_{s}=u_{s}^{h}, w_{s}=w_{s}^{h}},
\end{aligned}
$$

all of which depend on the nominal gap thickness $d_{z}$, the shear displacement $d_{x}$, and the parameters determining the interaction potential $U$. It is noted that the quantities $\mathcal{Y}_{s}, \mathcal{Y}_{\text {st }}$, and $\mathcal{Y}_{t}$ can be interpreted as the components of an interaction stiffness tensor

$$
\mathcal{Y}=\left(\begin{array}{ll}
\mathcal{Y}_{s} & \mathcal{Y}_{\mathrm{st}} \\
\mathcal{Y}_{\mathrm{st}} & \mathcal{Y}_{t}
\end{array}\right)
$$

which generalizes the scalar interaction stiffness defined in Refs. 13 and 14. These quantities have dimensions of force per unit volume, and characterize how the interaction force changes with the displacements $u_{s}$ and $w_{s}$. The specific dependence of $\mathcal{Y}$ on $d_{z}$ and $d_{x}$ depends on the exact from of the

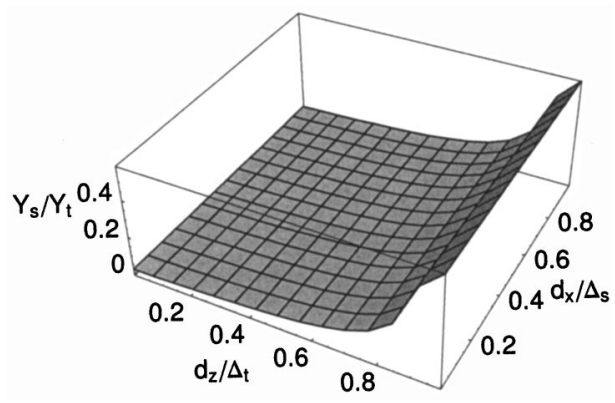

FIG. 3. Ratio of $\mathcal{Y}_{s}$ and $\mathcal{Y}_{t}$ as a function of $d_{x}$ and $d_{z}$. The cohesive potential given in Ref. 17 is used to generate this plot.

interaction potential. In general as $d_{z}$ increases (for example, with $d_{x}=0$ ), it is expected that all the elements of $\mathcal{Y}$ fall as some power of $d_{z}$. Similarly, for a fixed $d_{z}, \mathcal{Y}$ may be expected to fall with $d_{x}$.

Relative magnitudes of $\mathcal{Y}_{t}$ and $\mathcal{Y}_{s}$ can be obtained by considering, for example, the cohesive potential given in Ref. 17. This cohesive potential is such that, for $d_{x}=0$, as $d_{z}$ is increased, the normal stress $F_{z}$ reaches a maximum when $d_{z}=\Delta_{t}$. Similarly, on increasing $d_{x}$ with $d_{z}=0$, the shear stress $F_{x}$ attains a maximum at $d_{x}=\Delta_{s}$. Considering $\Delta_{s}$ $\approx 10 \Delta_{t}$, i.e., assuming that the forces fall much faster with the normal separation, it is obtained that $0.1 \mathcal{Y}_{t} \leqslant \mathcal{Y}_{s} \leqslant 0.5 \mathcal{Y}_{t}$. A plot of $\mathcal{Y}_{s} / \mathcal{Y}_{t}$ is shown in Fig. 3.

To obtain conditions for the existence of instability fields $\left(u_{s}^{p}, w_{s}^{p}\right)$, Eq. (7) is substituted into Eq. (1) following which the functional Eq. (1) is minimized. This exercise results in

$$
\begin{gathered}
\sigma_{x z s}^{p}=\mathcal{Y}_{s} u_{s}^{p}+\mathcal{Y}_{s t} w_{s}^{p}, \\
\sigma_{z z s}^{p}=\mathcal{Y}_{s t} u_{s}^{p}+\mathcal{Y}_{t} w_{s}^{p},
\end{gathered}
$$

where $\sigma_{x z s}^{p}=\sigma_{x z}^{p}(x, 0)$ and $\sigma_{z z s}^{p}=\sigma_{z z}^{p}(x, 0)$ are stresses due to the perturbation fields $\left(u^{p}, w^{p}\right)$ along the surface of the film.

Further, it is assumed that the instability fields have the form

$$
u_{s}^{p}(x)=\alpha e^{i k x}, \quad w_{s}^{p}(x)=\beta e^{i k x}
$$

to mimic wave-like perturbations of the surface, where $k$ is the wave number of the instability field and $\alpha$ and $\beta$ are complex numbers yet to be determined. The function $u_{s}^{p}$ describes the shear displacement perturbation of the surface, while $w_{s}^{p}$ describes the normal displacement perturbation. The conditions for existence of nontrivial instability fields can now be obtained by insisting that the complex numbers $\alpha$ and $\beta$ not vanish simultaneously. This is accomplished by use of the condition Eq. (10). The terms on the left side of Eq. (10) are the stresses along the surface of the film corresponding to surface displacements in Eq. (11). It can be shown that (by modification to the formulas given in the Appendix of Ref. 23 where the condition of prescribed shear displacement is used instead of a vanishing shear stress) for the given surface displacements in Eq. (11), the stress components along the surface are given by

$$
\sigma_{x z s}^{p}=\frac{\mu}{h}\left[S_{x z}^{x}(h k) \alpha-i S_{x z}^{z}(h k) \beta\right] e^{i k x},
$$




$$
\sigma_{z z s}^{p}=\frac{\mu}{h}\left[i S_{z z}^{x}(h k) \alpha+S_{z z}^{z}(h k) \beta\right] e^{i k x}
$$

where

$$
\begin{aligned}
& S_{x z}^{x}(q)=\frac{2 q[\sinh (2 q)-2 q]}{\cosh (2 q)-2 q^{2}-1}, \\
& S_{x z}^{z}(q)=S_{z z}^{x}(q)=\frac{4 q^{3}}{\cosh (2 q)-2 q^{2}-1}, \\
& S_{z z}^{z}(q)=\frac{2 q[\sinh (2 q)+2 q]}{\cosh (2 q)-2 q^{2}-1} .
\end{aligned}
$$

Again, $S_{x z}^{x}(q), S_{x z}^{z}(q)$, and $S_{z z}^{z}(q)$ may be interpreted as the components of a positive definite Hermitian tensor $\mathcal{S}$

$$
\mathcal{S}(q)=\left(\begin{array}{cc}
S_{x z}^{x}(q) & -i S_{x z}^{z}(q) \\
i S_{x z}^{z}(q) & S_{z z}^{z}(q)
\end{array}\right) .
$$

The condition for the existence of nontrivial instability fields can be obtained by substitution of Eq. (12) into Eq. (10), resulting in

$$
\left(\begin{array}{cc}
S_{x z}^{x}(q)-Y_{s} & -i S_{x z}^{z}(q)-Y_{s t} \\
i S_{x z}^{z}(q)-Y_{s t} & S_{z z}^{z}(q)-Y_{t}
\end{array}\right)\left(\begin{array}{l}
\alpha \\
\beta
\end{array}\right)=\left(\begin{array}{l}
0 \\
0
\end{array}\right),
$$

where

$$
Y_{s}=\frac{h \mathcal{Y}_{s}}{\mu}, \quad Y_{\mathrm{st}}=\frac{h \mathcal{Y}_{\mathrm{st}}}{\mu}, \quad Y_{t}=\frac{h \mathcal{Y}_{t}}{\mu}
$$

and

$$
q=h k
$$

are nondimensional values of interaction stiffness components and the wave number, respectively. The condition for the existence of nontrivial instability fields is obtained as

$$
\begin{aligned}
\operatorname{det}\left(\mathcal{S}-\frac{h}{\mu} \mathcal{Y}\right)= & {\left[S_{x z}^{x}(q)-Y_{s}\right]\left[S_{z z}^{z}(q)-Y_{t}\right] } \\
& -\left\{\left[S_{x z}^{z}(q)\right]^{2}+Y_{\mathrm{st}}^{2}\right\}=0 .
\end{aligned}
$$

The condition Eq. (20) may be understood as follows. The additional energy in the system due to the instability fields Eq. (11) per unit length of the film (in the $x$ direction) can be shown to be equal to

$$
\Delta \Pi=\frac{1}{2}\left(\begin{array}{ll}
\alpha^{*} & \beta^{*}
\end{array}\right)\left(\frac{\mu}{h} \mathcal{S}(q)-\mathcal{Y}\right)\left(\begin{array}{l}
\alpha \\
\beta
\end{array}\right),
$$

where ()$^{*}$ denotes the complex conjugate. For a given $\mathcal{Y}$, there will be no instability if

$$
\left(\frac{\mu}{h} \mathcal{S}(q)-\mathcal{Y}\right)
$$

is positive definite. It is therefore necessary to obtain the condition when the tensor

$$
\left(\frac{\mu}{h} \mathcal{S}(q)-\mathcal{Y}\right)
$$

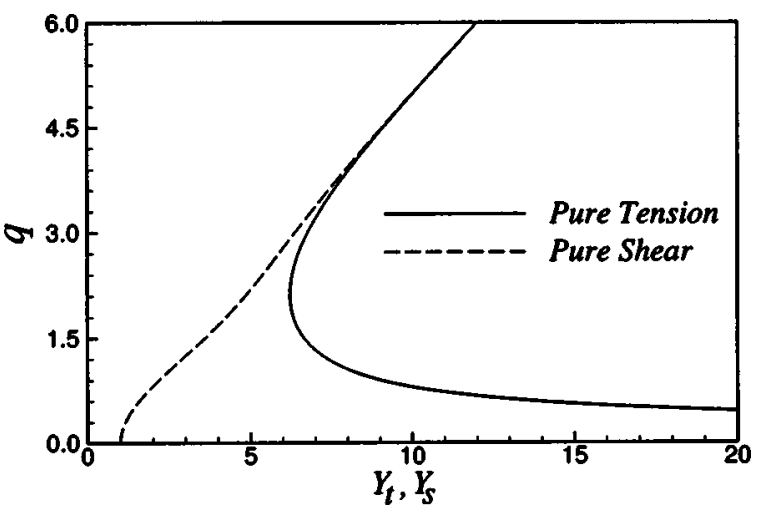

FIG. 4. Possible instability modes for pure tension and pure shear interactions.

loses its positive definite character, i.e., at least one of its eigenvalues vanishes, a condition that is equivalent to Eq. (20) (since the determinant is the product of the eigenvalues of the tensor). Evidently, if the tensor $\mathcal{Y}$ is negative definite, instability is not possible; expressed mathematically, there is no possibility of instability if $\mathcal{Y}_{s}+\mathcal{Y}_{t}<0$ and $\mathcal{Y}_{s} \mathcal{Y}_{t}>\mathcal{Y}_{\mathrm{st}}^{2}$.

The condition Eq. (20) can be further simplified as

$$
e(q) Y_{t}+f(q) Y_{s}+\left(Y_{s} Y_{t}-Y_{\mathrm{st}}^{2}\right) g(q)+p(q)=0,
$$

where

$$
\begin{aligned}
& e(q)=-2 q[\sinh (2 q)-2 q], \\
& f(q)=-2 q[\sinh (2 q)+2 q], \\
& g(q)=\cosh (2 q)-2 q^{2}-1, \\
& p(q)=4 q^{2}\left[1+2 q^{2}+\cosh (2 q)\right] .
\end{aligned}
$$

If the interaction potential does not depend on $u_{s}$, then $Y_{s}$ $=Y_{s t}=0$ and Eq. (22) reduces to

$$
\frac{2 q\left[1+2 q^{2}+\cosh (2 q)\right]}{\sinh (2 q)-2 q}=Y_{t},
$$

which is precisely the result of Refs. 13 and 14. This type of interaction will be referred to as a "pure tension interaction." A second case of interest, which will be used in Sec. III B, called the "pure shear interaction," is when the interaction potential depends only on $u_{s}$ (and not on $w_{s}$, hence $Y_{t}$ $\left.=Y_{\text {st }}=0\right)$. In this case Eq. (22) reduces to

$$
\frac{2 q\left[1+2 q^{2}+\cosh (2 q)\right]}{\sinh (2 q)+2 q}=Y_{s} .
$$

Relations (28) and (27) are pictorially depicted in Fig. 4.

For the case of pure tension interaction, the critical value of $Y_{t}$ and the corresponding wave number of the critical mode are given by

$$
Y_{t}^{c}=6.22, \quad q_{t}^{c}=2.12
$$

and those for the pure shear case are given by

$$
Y_{s}^{c}=1, \quad q_{s}^{c}=0 .
$$

The value $Y_{t}^{c}$ is the smallest possible value of the interaction stiffness that will trigger an instability in the system. A similar meaning is associated with $Y_{s}^{c}$ for the case of shear. These 
relations will be used in the discussion of the general case, which is considered in Sec. III. The condition Eq. (29) may be understood in the following manner. Instability sets in when $Y_{t}$ exceeds a critical value, i.e., when a critical distance $d_{z}^{c}$ is attained. Also, the dimensionless wavelength of the pattern that emerges given by $\lambda_{c}=2 \pi / q_{t}^{c}$ is independent of the magnitude of the interactions.

\section{RESULTS AND DISCUSSION}

Attention is now focused on the discussion of the general case where $Y_{t}, Y_{s}$, and $Y_{\text {st }}$ are all nonzero. An important point evident from Eq. (22) is that instabilities are not affected by the sign of $Y_{\text {st }}$, consequently, only positive values of $Y_{\text {st }}$ need to be considered. To obtain the dependence of the instability modes (described by the wave number $q$ ), the following strategy is adopted. A parameter $\eta$ (which can take on any real value) is introduced such that

$$
Y_{s}=\eta Y_{t} .
$$

The parameter $\eta$, which is akin to a "friction coefficient," is a measure of the relative strengths of $Y_{t}$ and $Y_{s}$, the tensile interaction stiffness and the shear interaction stiffness. A small value of $\eta$ implies that the tensile part of the interaction is dominant, and a large value (in magnitude) of $\eta$ implies that the interaction is dominated by the shear. A related quantity that allows for a graphical interpretation of $\eta$ is defined as

$$
\theta=\tan ^{-1} \eta=\tan ^{-1}\left(\frac{Y_{s}}{Y_{t}}\right),
$$

which may be interpreted as the angle made by the line joining the point $\left(Y_{t}, Y_{s}\right)$ to the origin in the $Y_{t}-Y_{s}$ parameter space. Although the results are presented for all values of $Y_{t}$ and $Y_{s}$, the values relevant to experiments fall in a narrow range of $0.1 Y_{t} \leqslant Y_{s} \leqslant 0.5 Y_{t}$ as discussed in the previous section using the cohesive potentials given in Ref. 17.

On substitution of Eq. (31), Eq. (22) reduces to

$$
\eta g(q) Y_{t}^{2}+[e(q)+\eta f(q)] Y_{t}+\left[p(q)-Y_{\mathrm{st}}^{2} g(q)\right]=0 .
$$

This equation, a quadratic in $Y_{t}$, is solved to obtain two roots:

$$
Y_{t}^{(1)}=\frac{-[e(q)+\eta f(q)]+\sqrt{[e(q)+\eta f(q)]^{2}-4 \eta g(q)\left[p(q)-Y_{\mathrm{st}}^{2} g(q)\right]}}{2 \eta g(q)}
$$

and

$$
Y_{t}^{(2)}=\frac{-[e(q)+\eta f(q)]-\sqrt{[e(q)+\eta f(q)]^{2}-4 \eta g(q)\left[p(q)-Y_{\mathrm{st}}^{2} g(q)\right]}}{2 \eta g(q)} .
$$

For a given value of $\eta$ and $Y_{\mathrm{st}}$, the possibility of real values of $q$ satisfying Eqs. (34) and (35) is investigated separately as $Y_{t}^{(1)}$ and $Y_{t}^{(2)}$ are, respectively, varied. The smallest value of $Y_{t}^{(1)}\left(Y_{t}^{(2)}\right)$ for which there exist real values of $q$ satisfying Eqs. (34) and (35) is taken to be the critical tensile interaction stiffness $Y_{t}^{1 c}\left(Y_{t}^{2 c}\right)$ and the corresponding critical shear interaction stiffness is $Y_{s}^{1 c}\left(Y_{s}^{2 c}\right)$ is obtained by multiplying $Y_{t}^{1 c}\left(Y_{t}^{2 c}\right)$ with $\eta$. The value of the $q$ that satisfies Eqs. (34) and (35) for $Y_{t}^{1 c}\left(Y_{t}^{2 c}\right)$ is called the critical mode $q^{1 c}\left(q^{2 c}\right)$.

\section{A. Results}

The two cases implied by Eqs. (34) and (35) are discussed separately so as to clearly understand the nature of the instability in the two cases. The physical meaning of the two roots will be discussed in detail after the description of the solution. It suffices to mention here that the first root Eq. (34) is relevant to the contact experiments, while the second root Eq. (35) is useful in the understanding of peeling experiments.

The two roots represent two "surfaces" of instability solutions. For given values of $Y_{t}$ and $Y_{s}$, if there are real values of $q$ which satisfy either of Eqs. (34) or (35), then this state is unstable. Results are presented in such a way that the values of $q$ are plotted as a function of $Y_{t}$ and $Y_{s}$ [see Eqs.
(34) and (35)]. The two roots represent distinct surfaces. The calculations indicate that there are two distinct surfaces (given by the two roots) only in the first quadrant of the $Y_{t}$ $-Y_{s}$ space. In the second and fourth quadrants, solutions obtained from the two roots are a continuation of the surface obtained from the first root in the first quadrant. Due to this reason, the results in the second and fourth quadrant are discussed along with the results of Eq. (34) in the first quadrant.

\section{First root}

The results of the procedure to determine possible instability modes outlined in the preceding paragraphs applied to Eq. (34) are graphically depicted in Fig. 5. Instability modes are shown for various values of $Y_{\mathrm{st}}$.

The main features are as follows:

(i) For small values of $Y_{\text {st }}$, there are instabilities possible when the values of $\theta$ [as defined in Eq. (32)] are in the range $-\pi / 2 \leqslant \theta \leqslant \pi$. The number of instability modes for the unstable values of $Y_{s}$ and $Y_{t}$ are: one in $\pi / 2 \leqslant \theta \leqslant \pi$; one, two or three in the first quadrant $0 \leqslant \theta \leqslant \pi / 2$ of the $Y_{t}-Y_{s}$ plane; and one or two in the fourth quadrant $-\pi / 2 \leqslant \theta \leqslant 0$. There are no instabilities possible in the third quadrant for small values of $Y_{\mathrm{st}}$.

(ii) For larger values of $Y_{\text {st }}$ instabilities are possible even in the third quadrant of the $Y_{t}-Y_{s}$ plane. The smallest 

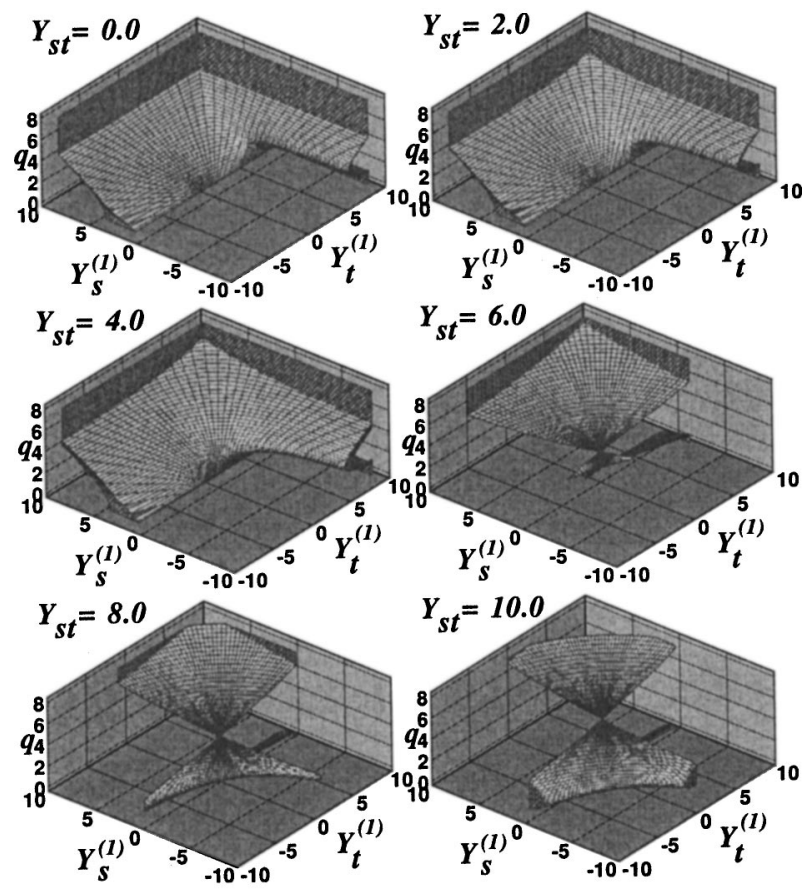

FIG. 5. Instability modes as a function of the interaction stiffnesses for the first root Eq. (34). The surfaces depict the dependence of the wave number of instability mode(s) on the values of $Y_{t}$ and $Y_{s}$, for different values of $Y_{\text {st }}$.

value of $Y_{\mathrm{st}}$ for which this becomes possible is $Y_{\mathrm{st}}^{m}=5.82$; for this value of $Y_{\text {st }}$, the critical tension interaction stiffness is $Y_{t}^{1 c}=0$ and the critical shear interaction stiffness is $Y_{s}^{1 c}=0$.

(iii) For values of $Y_{\mathrm{st}}>Y_{\mathrm{st}}^{m}$ there are values of $\eta$ that lie between $\eta_{a}$ and $\eta_{b}$, i.e., $\eta^{a} \leqslant \eta \leqslant \eta^{b}$ (cf. Fig. 6) for which there are values of $q$ such that the discriminant of Eq. (34) is negative. For these values of $\eta$, and for the values of $q$ for which the discriminant is negative, there are no values of $Y_{t}$ for which these values of $q$ are the instability modes. The complete set of values of $\eta$ and $q$ that do not have instabilities depends on $Y_{\text {st }}$. A plot of such values is given in Fig. 7 for several values of $Y_{\mathrm{st}}>Y_{\mathrm{st}}^{m}$.

Figure 8 shows a plot of the critical tensile interaction stiffness as a function of $\eta$, for various values of $Y_{\mathrm{st}}$. It is

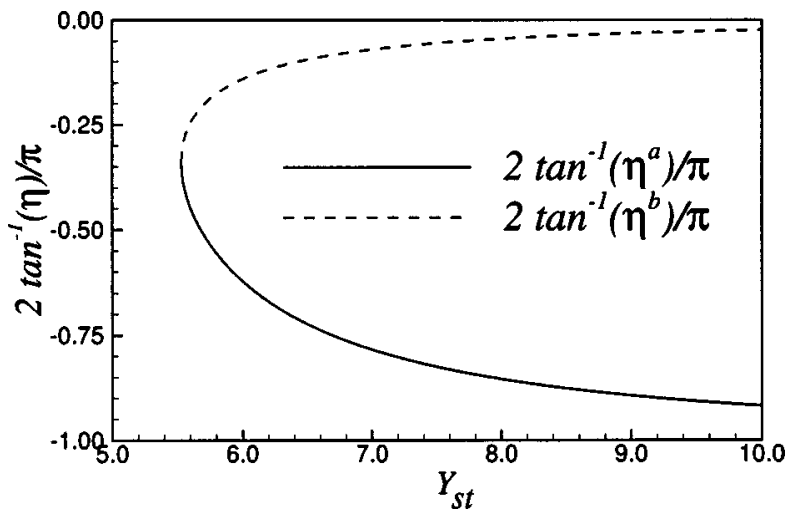

FIG. 6. Range of $\eta$ as a function of $Y_{\text {st }}$ for which the discriminant of Eq. (34) is negative for some values of $q$. When $\eta$ lies between $\eta_{a}$ and $\eta_{b}$, there are values of $q$ for which the discriminant of Eq. (34) is negative. These values of $q$ are not instability modes for any value of $Y_{t}^{1}$.

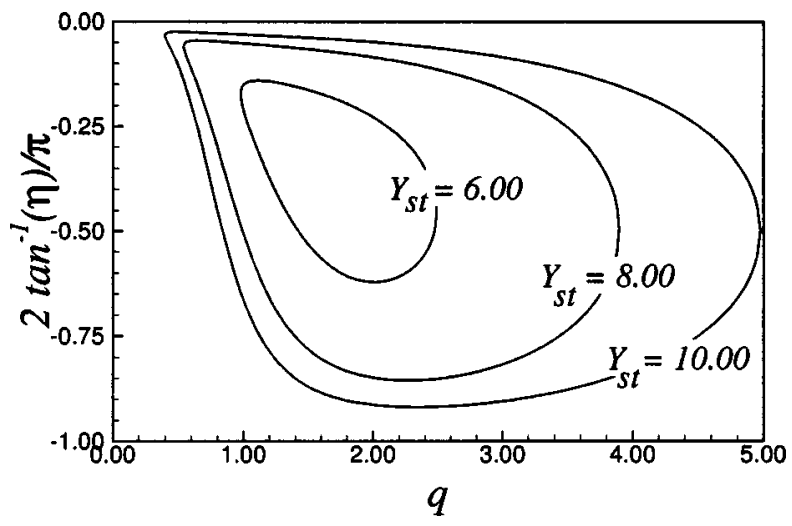

FIG. 7. Points inside the closed curves are the values of $\eta$ and $q$ for which $q$ is not an instability mode for any value of $Y_{t}^{(1)}$.

seen that for values of $Y_{\mathrm{st}}$ less than $Y_{\mathrm{st}}^{m}, Y_{t}^{1 c}$ falls with increasing $\eta$. However, the trend is reversed at higher values of $Y_{\text {st }}$. Figure 9 shows a similar plot of the critical shear interaction stiffness as a function of $\eta$. The trend as a function of $\eta$ is opposite to that of $Y_{t}^{1 c}$. The stability line in the $Y_{t}$ $-Y_{s}$ parameter space is shown in Fig. 10; regions to the left of the lines are regions where the homogeneous solution is stable, while those to the right of the lines are regions where instabilities are possible. It is noted that the boundary separating the stable and unstable regions in the $Y_{s}-Y_{t}$ space has "kinks" in the first quadrant, especially for values of $Y_{\text {st }}$ smaller than $Y_{\mathrm{st}}^{m}$. The dependence of the critical mode $q^{1 c}$ on $\eta$ is shown in Fig. 11. For small values of $Y_{\text {st }}$, the wave number of the critical mode falls monotonically as a function of $\eta$. Indeed, for small $Y_{\mathrm{st}}$ (for example, $Y_{\mathrm{st}}=0,2$ ), the critical mode changes discontinuously from a finite value to zero at a particular value of $\eta$. Figure 12 shows a plot of the values of $\eta$ at which the jump occurs (called $\eta_{J}$ ) as a function of $Y_{\mathrm{st}}$. The value of jump in the critical wave number $q_{J}^{1 c}$ is also plotted in the same graph. The value of $\eta_{J}$ increases with $Y_{\text {st }}$ and the value of the jump in the critical wave number decreases; at values of $Y_{\text {st }}$ greater than about 4, the discontinuity in the wave number as a function of $\eta$ vanishes (Fig. 11). For values of $Y_{\mathrm{st}}$ larger than $Y_{\mathrm{st}}^{m}, q^{1 c}$ is an increasing function of $\eta$, i.e., the trend is reversed.

As discussed in the previous section, the condition for the onset of instability is the loss of positive definiteness of

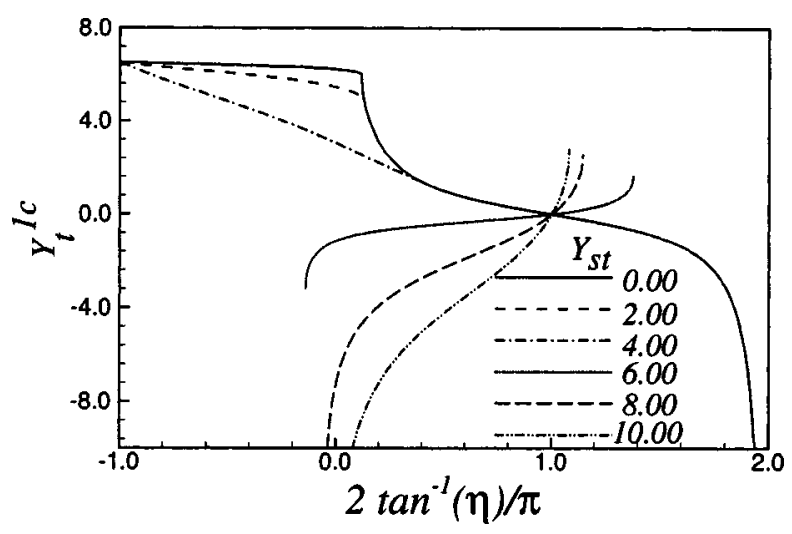

FIG. 8. Critical tensile interaction stiffness $Y_{t}^{1 c}$ as a function of $\eta$. 


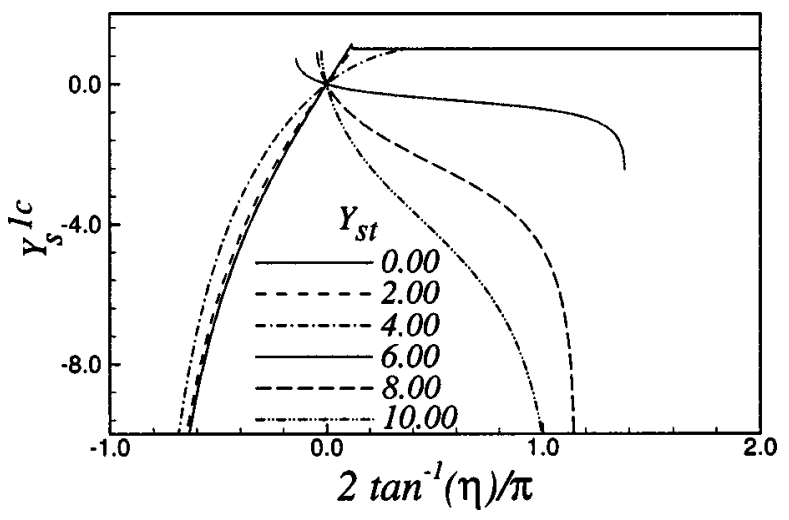

FIG. 9. Critical shear interaction stiffness $Y_{s}^{1 c}$ as a function of $\eta$.

the tensor, $(\mu / h) \mathcal{S}-\mathcal{Y}$ [Eq. (21), and surrounding arguments]. For small values of $Y_{\text {st }}$, the relative magnitudes of $Y_{t}$ and $Y_{s}$ govern the nature of the instability. When $Y_{s}$ $\ll Y_{t}$, i.e., for very small values of $\eta$, the instability is governed by $Y_{t}$, and the critical tensile interaction stiffness is close to that given in Eq. (27). Also, for large values of $\eta$, the instability is governed entirely by the shear interaction stiffness. When $Y_{\mathrm{st}} \leq 4$, the transition from the tension dominated response to the shear dominated response occurs drastically. The signature of this phenomenon is the discontinuous change of $q^{1 c}$ from a finite value (tension dominated) to zero (shear dominated). This is also the reason for the "kinks" in the stability boundary in the $Y_{t}-Y_{s}$ parameter space. This is further confirmed in a plot of the ratio of $(|\alpha| /|\beta|)^{1 c}$ shown in Fig. 13, i.e., the ratio of the magnitudes of the shear displacement of the surface to the normal displacement of the surface. It is evident that for values of $Y_{\mathrm{st}} \leqslant 4$, there is a drastic increase in $(|\alpha| /|\beta|)^{1 c}$ at $\eta=\eta_{J}$, indicating a transition from tension dominated to shear dominated behavior. When $Y_{\mathrm{st}}<Y_{\mathrm{st}}^{m}$, it is seen that $q^{1 c}$ increases as $\eta$ tends $-\infty$ (or $\theta$ approaches $-\pi / 2$ ), and approaches a limiting value of about 2.5. In this regime, $Y_{s}<0$, i.e., shear therefore contributes to increase the additional energy Eq. (21) in the system. This increase in energy due to shear can be offset by choosing a small value of $(|\alpha| /|\beta|)^{1 c}$ (i.e., normal displacement

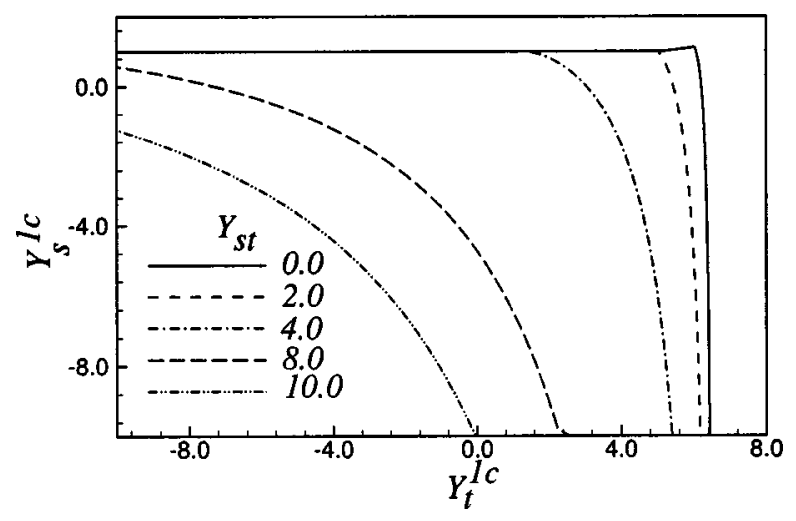

FIG. 10. Stability lines in $Y_{t}-Y_{s}$ parameter space for different values of $Y_{\text {st }}$. The homogeneous solution is stable in the region to the left of the lines, and unstable to the right.

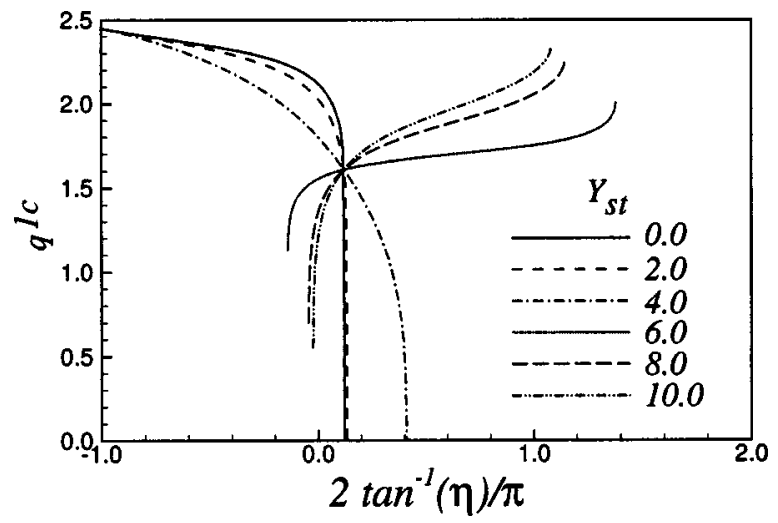

FIG. 11. Critical wavenumber $q^{1 c}$ as a function of $\eta$.

dominated instability) such that the reduction in energy Eq. (21) from normal displacements is large enough.

For values of $Y_{\mathrm{st}}>Y_{\mathrm{st}}^{m}$, the arguments used above cannot be applied directly since the off diagonal term in the tensor $\mathcal{Y}$ affects the results in a strong manner. The main point to be noted is that as $\eta$ increases (i.e., $Y_{s}$, though negative, increases in magnitude) and $Y_{t}$ decreases in magnitude (although it increases, numerically). Both of these contribute to the increase in additional energy Eq. (21) (i.e., they make positive contributions). Consequently, with the increase of $\eta$, i.e., the interaction term is dominated by the shear interaction stiffness (in magnitude), and the deformation mode is normal displacement dominated. This is evident in Fig. 11, where the critical wave number increases with increase of $\eta$. In addition, the ratio $(|\alpha| /|\beta|)^{1 c}$ decreases with increasing $\eta$, indicating that the deformation mode is dominated by the normal displacement $\beta$ (evident from curves for $Y_{\mathrm{sc}}=6,8$, 10, in Fig. 13).

\section{Second root}

A result uncovered in this analysis is the presence of a second root. As discussed above, this root occupies a distinct surface only in the first quadrant. Instability modes arising from the second root Eq. (35) are shown in Fig. 14 for various values of $Y_{\mathrm{st}}$. It is evident that there are two instability modes for unstable values of $Y_{t}$ and $Y_{s}$ except along the stability boundary where they are identical.

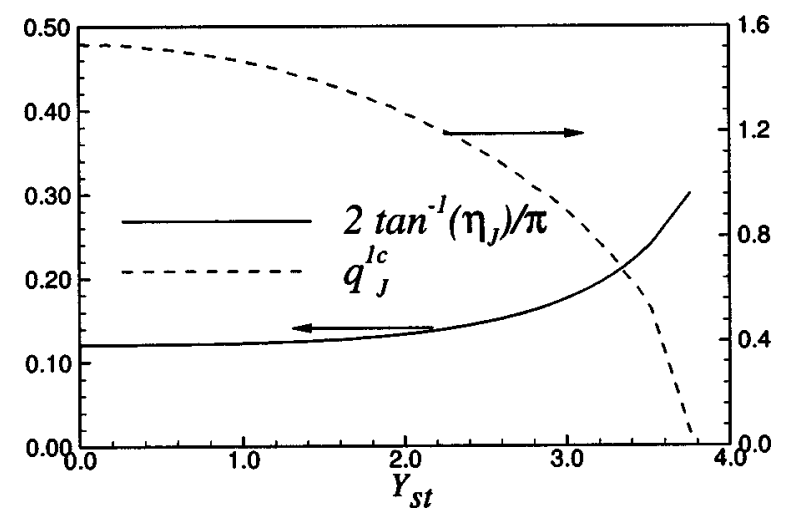

FIG. 12. Dependence of $\eta_{J}$ and $q_{J}^{1 c}$ on $Y_{\mathrm{st}}$. 


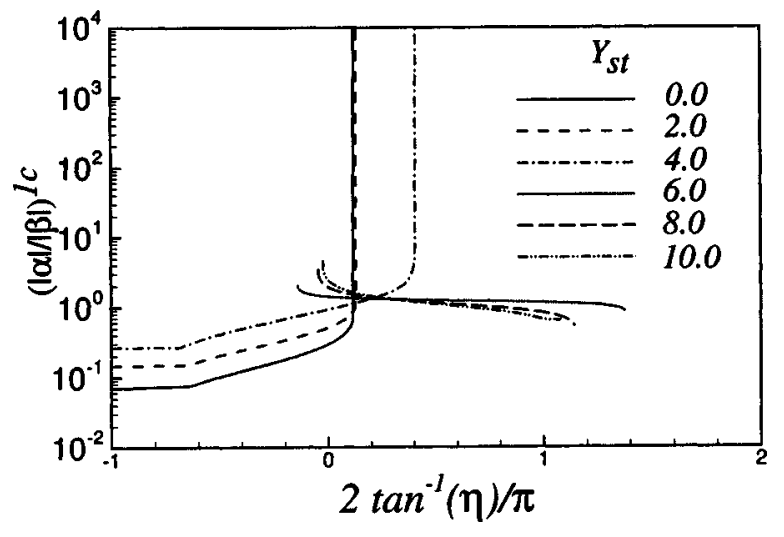

FIG. 13. Dependence of the ratio of relative magnitudes of shear and normal displacements $(|\alpha| /|\beta|)^{1 c}$ on $\eta$, for various values of $Y_{\text {st }}[\alpha$ and $\beta$ are as defined in Eq. (11)].

The dependence of the critical tensile interaction stiffness $Y_{t}^{2 c}$ on $\eta$ is shown in Fig. 15 where $Y_{t}^{2 c}$ decreases with increasing $\eta$ for all values of $Y_{\mathrm{st}}$ and attains a limiting value of 6.52 independent of the value of $Y_{\text {st }}$ as $\eta \rightarrow \infty$. The shear interaction stiffness increases with increasing $\eta$ as shown in Fig. 16. For small values of $\eta, Y_{s}^{2 c}$ is independent of $Y_{\mathrm{st}}$ and has a value of about 4.03 (Fig. 17). Figure 18 shows the dependence of the critical wave number $q^{2 c}$ on $\eta$; a surprising aspect is that $q^{2 c}$ increases with increase in $\eta$ (which may be contrasted with the behavior of $q^{1 c}$ ). A further interesting aspect is shown in Fig. 19 where the ratio of the relative magnitudes of shear and normal displacements is plotted. It is seen that for small values of $\eta$, the deformation in the film is shear displacement dominated, while at large values of $\eta$, the deformation is normal displacement dominated.

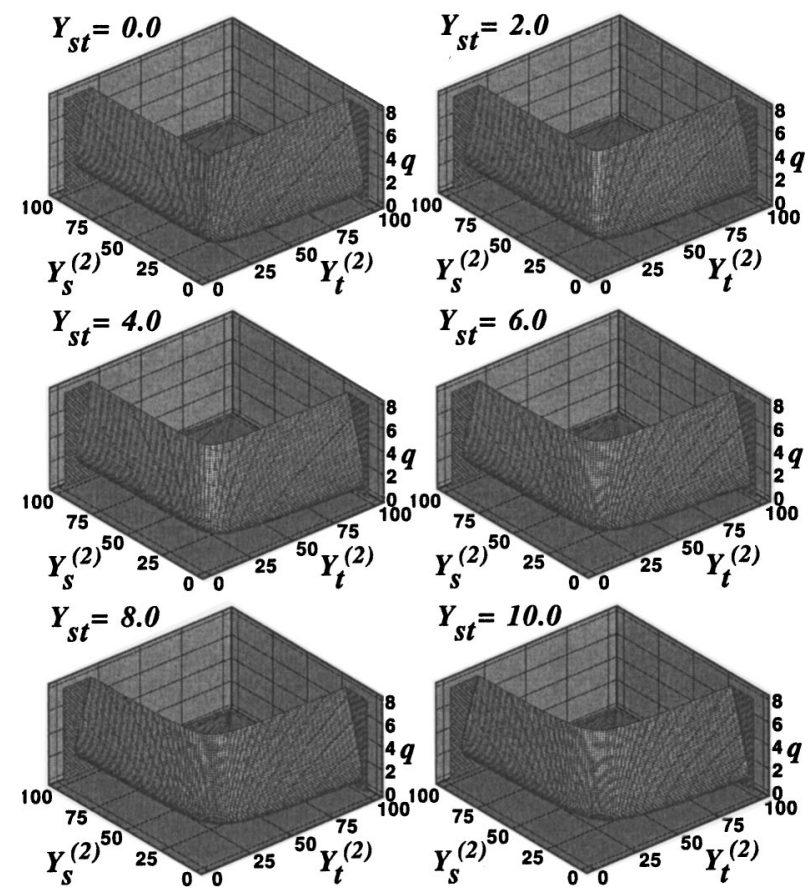

FIG. 14. Instability modes as a function of the interaction stiffnesses for the second root Eq. (35). The surfaces depict the dependence of the wave number of instability mode(s) on the values of $Y_{t}$ and $Y_{s}$, for different values of $Y_{\mathrm{st}}$.

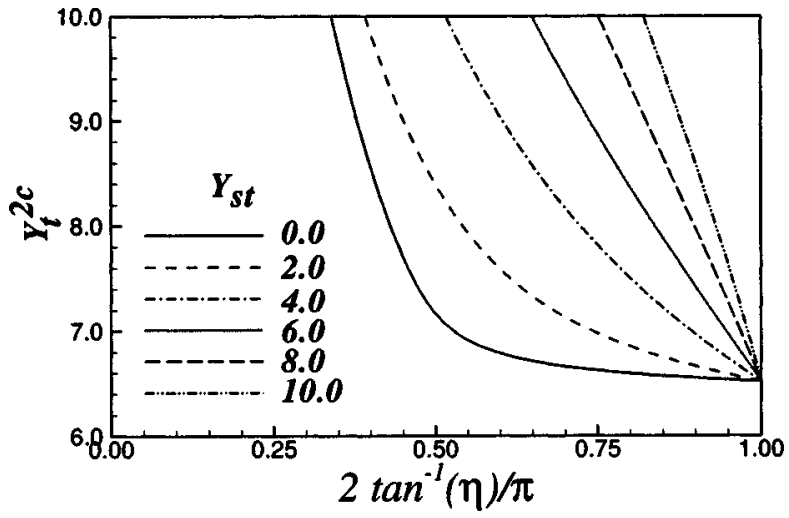

FIG. 15. Critical tensile interaction stiffness $Y_{t}^{2 c}$ as a function of $\eta$.

The underlying physics of these results can be understood by noting the crucial difference between the conditions for the onset of instability in the case of the second root as compared to the first root. For small values of $Y_{\text {st }}$ and small positive values of $\eta$, the instability in the case of the first root is controlled by the tensile interaction stiffness $Y_{t}^{1 c}$, the critical value of which is about 6.22 as given in Eq. (27). On the other hand, the critical value of $Y_{t}^{2 c}$ for these values of $\eta$ is very large. Thus, further energy reduction in Eq. (21) can be achieved by making the shear component of the deformation large compared to the normal component. This explains the large value of $(|\alpha| /|\beta|)^{2 c}$ for small values of $\eta$. The same argument also explains the fall of the critical wave number on reduction of $\eta$. Indeed, if the deformation is to be shear displacement dominated, then the elastic energy cost can be reduced [making Eq. (21) more negative] if the wave number of the instability is made smaller since for a given amplitude of shear displacement, energy is lower for smaller wave numbers. Of course, smaller wave numbers mean higher elastic energy cost when viewed in terms of normal displacements. Thus, the critical wave number is larger than zero, but smaller than 2.12, which is the case in Eq. (27). The effect of $Y_{\mathrm{st}}$ can be understood as follows; for large values of $Y_{\mathrm{st}}$, there is a corresponding increase in $Y_{t}^{2 c}$ and $Y_{s}^{2 c}$ so that the tensor $\mathcal{Y}$ remains positive definite. Another important point is that there is very little effect of $Y_{\text {st }}$ on the critical wave number, especially for values of $Y_{\text {st }}$ larger than zero. This may be understood from the fact that since the critical values

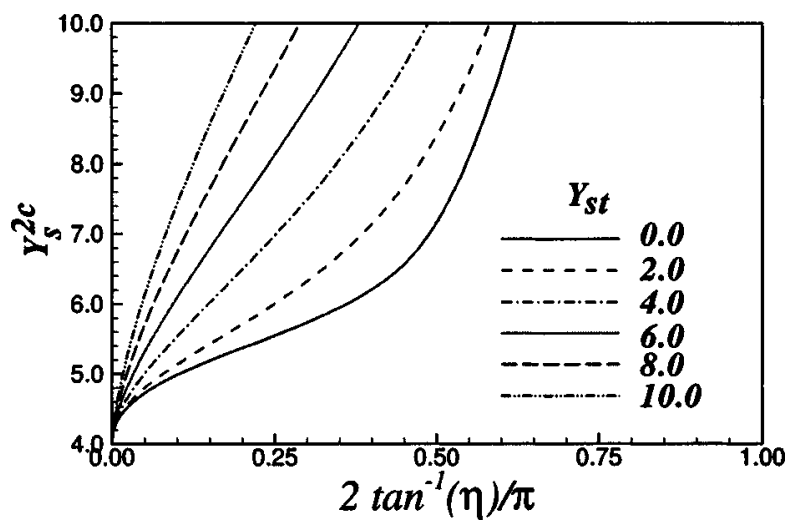

FIG. 16. Critical shear interaction stiffness $Y_{s}^{2 c}$ as a function of $\eta$. 


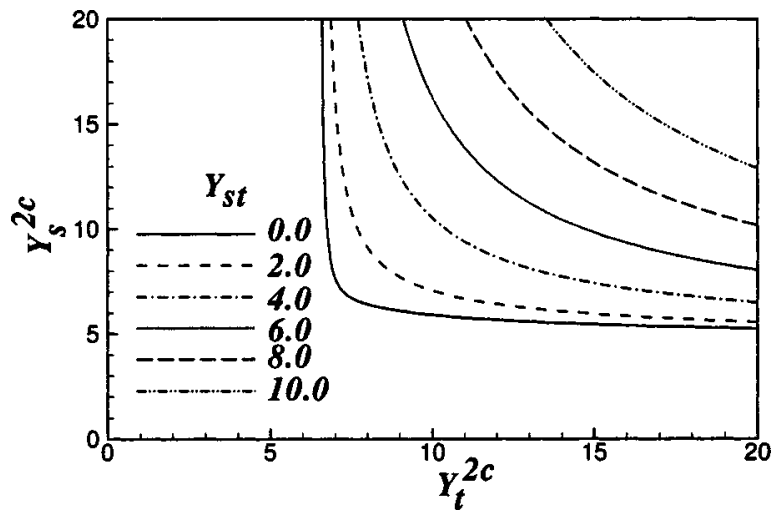

FIG. 17. Stability lines in $Y_{t}-Y_{s}$ parameter space for different values of $Y_{s t}$ for the second root Eq. (35). The homogeneous solution is stable in the region to the left of the lines, and unstable to the right.

of $Y_{t}^{2 c}$ and $Y_{s}^{2 c}$ are numerically much larger than $Y_{\text {st }}$ (except in the region where $\eta \approx 1.0), Y_{\text {st }}$ does not contribute significantly in Eq. (21).

The results presented here may be used in the following manner to arrive at the wavelengths of the instability under different conditions. For any given configuration the values of $Y_{t}, Y_{s}$, and $Y_{\text {st }}$ are calculated from Eqs. (8) and (18). If the value of $Y_{s}<4$, then there is no possibility of the second root, and in such cases the instability is dominated by the first root for all values of $Y_{\mathrm{st}}$, and the results for the critical wave number can be obtained from Fig. 11. On the other hand if conditions are such that $Y_{s}>4$ and $Y_{t}>6.5$, then the relevant root for all values of $Y_{\mathrm{st}}$ is the second root; the wavelength of the resulting pattern can be obtained from Fig. 18 .

\section{B. Discussion}

The results presented in the previous section uncover the rich and complex nature of the elastic instability in soft films with interactions that depend both on normal and shear displacements. Two points, in the main, require discussion. They are the significance of the two roots, and the dependence of the critical wave number on the interaction stiffness.

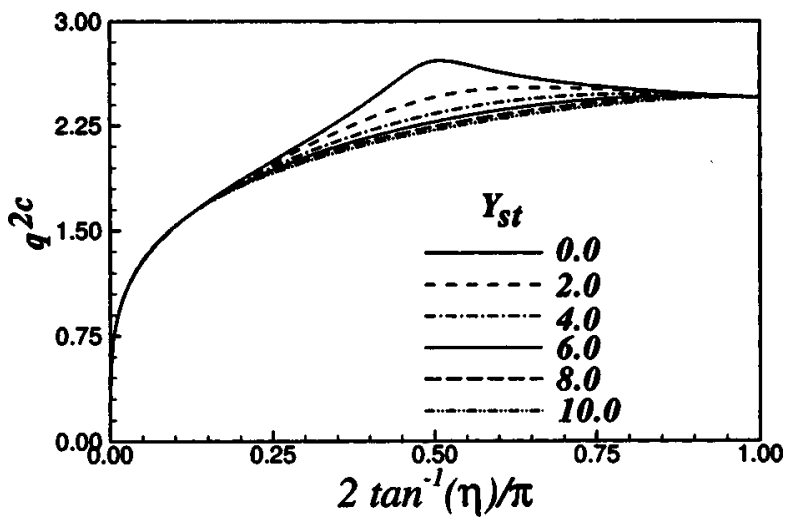

FIG. 18. Critical wave number $q^{2 c}$ as a function of $\eta$.

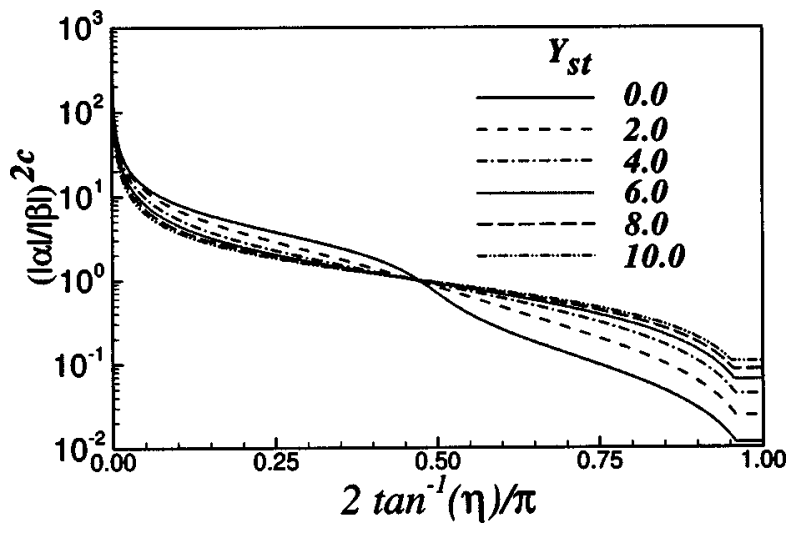

FIG. 19. Dependence of the ratio of relative magnitudes of shear and normal displacements $(|\alpha| /|\beta|)^{2 c}$ on $\eta$, for various values of $Y_{\text {st }}[\alpha$ and $\beta$ are as defined in Eq. (11)].

The results related to the first root indicate that the instability modes corresponding to this root occur when $Y_{t}$ is small in magnitude. This is indeed the case in a contact experiment where a flat contactor is bought in proximity of a soft film surface starting from an initially large separation. The shear interaction stiffness is much smaller compared to $Y_{s} \ll Y_{t}$ (also $\left.Y_{\mathrm{st}} \ll Y_{t}\right)$. Therefore, wavelength of the instability in the contact experiment is essentially described by Eq. (27). The wavelength of the instability is therefore independent of the magnitude and decay behavior of the interaction. Thus the theory presented in Refs. 13 and 14 applies well to the experiments of Ref. 11 which are contact type experiments.

In a "peeling" experiment (see Fig. 1), on the other hand, where a contactor initially in complete contact with the film is peeled, a curved flexible contactor related to open a "crack" along the contact region, thus engendering shear effects. The values of both $Y_{t}$ and $Y_{s}$ are thus comparably large in the initial stages of the peeling. Therefore, it is the instability indicated by the second root that will be triggered. Generally $0 \leqslant Y_{s} \lesssim Y_{t}$ in this regime (see Fig. 3), and thus the critical wave number of the instability $q^{2 c}$ lies between 1.5 and 2, i. e., the wavelength of the instability will be between $3 h$ and $4 h$. Another interesting point is that in this range, the critical wave number does not depend on $Y_{\text {st }}$, and therefore the wavelength is essentially independent of the interactions. Indeed, in the peeling type experiments of Ref. 10, the wavelength of the instability is found to be nearly equal to four times the film thickness.

It must be noted that the unperturbed state in the peel type experiments corresponds to a spatially inhomogeneous state, while the unperturbed state in the present analysis is a homogeneous plane strain state. Also, the contactor in the peel type experiments is flexible unlike the rigid contactor modeled here. Despite this, the analysis captures the essential physics for the following reason. The inhomogeneity is in the direction of the peel, i.e., in the direction of the crack propagation (cf. Fig. 1). Stresses do not vary along the direction of the meniscus (which is perpendicular to the plane direction of the crack propagation) in the unperturbed state. The direction of the meniscus is the direction of the $x$ axis in 
Fig. 2. The inhomogeneous state of stress along the direction of crack propagation serves to relieve the constraint (which is present in the contact type experiments) that brings into play the shear stresses at the meniscus. The present model, therefore, treats the effect of the spatial inhomogeneity in an approximate fashion by allowing for the effects of shear. The flexibility of the contactor is not captured by the present model and will be taken up for study in the future.

In summary, this article accounts for physics in the analysis of the adhesive instability by considering the coupled tension-shear effects in the interactions between the film and the contactor. The main finding of this article is that the wavelength of the instability is only slightly modified due to the presence of shear-tension coupling, and is in agreement with the trends seen in experiments. Important differences between contact type and peeling type experiments are uncovered.

\section{CONCLUSION}

This article develops on the analysis presented in Refs. 13 and 14 and extends the same to include coupled-shear tension interactions. The results presented here describe the nature of the instability over a wide range of interaction stiffnesses leading to a rather complex behavior of the critical wavelength as a function of the interaction. However, in the range of parameters actually attainable in experiments, it is seen that the essential features of the theory presented in Refs. 13 and 14 is preserved, in that the instability wavelength is essentially independent of the interaction and scales linearly with the film thickness. The key result of the present article is the demonstration of the existence of two different types of instabilities: one in contact type experiments and the other in peel type experiments. Also, the present analysis indicates that the wavelength of the instability in peel type experiments is expected to be larger than those in contact type experiments, as is also borne out in the experiments cited.

${ }^{1}$ R. J. Asaro and W. A. Tiller, Metall. Trans. 3, 1789 (1972).

${ }^{2}$ J. C. Ramirez, Int. J. Solids Struct. 25, 579 (1989).

${ }^{3}$ D. Srolovitz, Acta Metall. 37, 621 (1989).

${ }^{4}$ M. Grinfeld, J. Nonlinear Sci. 3, 35 (1993).

${ }^{5}$ J. A. Flaro, G. A. Lucadamo, L. B. Freund, M. Sinclair, R. Q. Twesten, and R. D. Hwang, Phys. Rev. Lett. 80, 4717 (1998).

${ }^{6}$ S. Herminghaus, K. Jacobs, K. Mecke, J. Bischof, A. Fery, M. Ibn-Elhaj, and S. Schlagowski, Science 282, 916 (1998).

${ }^{7}$ A. Sharma and R. Khanna, Phys. Rev. Lett. 81, 3463 (1998).

${ }^{8}$ G. Reiter, R. Khanna, and A. Sharma, Phys. Rev. Lett. 85, 1432 (2000).

${ }^{9}$ E. Schäffer, T. Thurn-Albrecht, T. P. Russel, and U. Steiner, Nature (London) 403, 874 (2000).

${ }^{10}$ A. Ghatak, M. K. Chaudhury, V. Shenoy, and A. Sharma, Phys. Rev. Lett. 85, 4329 (2000).

${ }^{11}$ W. Mönch and S. Herminghaus, Europhys. Lett. 53, 525 (2001).

${ }^{12}$ K. R. Shull, C. M. Flanigan, and A. J. Crosby, Phys. Rev. Lett. 84, 3057 (2000).

${ }^{13}$ V. Shenoy and A. Sharma, Phys. Rev. Lett. 86, 119 (2001).

${ }^{14}$ V. Shenoy and A. Sharma, J. Mech. Phys. Solids 50, 1155 (2002).

${ }^{15}$ Y. Sun and G. Beltz, J. Mech. Phys. Solids 42, 1905 (1994).

${ }^{16}$ A. Needleman, J. Mech. Phys. Solids 38, 21 (1990).

${ }^{17}$ X. P. Xu and A. Needleman, J. Mech. Phys. Solids 42, 1397 (1993).

${ }^{18}$ J. H. Rose, J. Ferrante, and J. R. Smith, Phys. Rev. Lett. 47, 675 (1981).

${ }^{19}$ C. Y. Hui, Y. Y. Lin, and C. Creton, J. Polym. Sci., Part B: Polym. Phys. 40, 545 (2002).

${ }^{20}$ M. Newby, B. Z. Chaudhury, and H. Brown, Science 269, 1407 (1995).

${ }^{21}$ C. Gui, M. Elwenspoek, N. Tas, and J. G. E. Gardeniers, J. Appl. Phys. 85, 7448 (1999).

${ }^{22}$ L. D. Landau and E. M. Lifshitz, Theory of Elasticity (Pergamon, Oxford, England, 1989).

${ }^{23}$ V. Shenoy and A. Sharma, Langmuir 18, 2216 (2002). 